Jean-Briac G. Prévost

Joost J. Nuyttens

Mischa S. Hoogeman

Johan J. Pöll

Lukas C. van Dijk

Peter M. T. Pattynama

\section{Endovascular coils as lung tumour markers in real-time tumour tracking stereotactic radiotherapy: preliminary results}

Received: 23 September 2007

Revised: 26 January 2008

Accepted: 17 February 2008

Published online: 4 April 2008

(C) The Author(s) 2008

J.-B. G. Prévost $(\bowtie) \cdot$ J. J. Nuyttens ·

M. S. Hoogeman · J. J. Pöll

Department of Radiation Oncology,

Erasmus Medical Center - Daniel den

Hoed Cancer Center,

Postbus 5201,

3008 AE Rotterdam, The Netherlands

e-mail: jean-briac.prevost@

uz.kuleuven.ac.be

Tel.: +32-16-347602

Fax: +32-16-347623

L. C. van Dijk - P. M. T. Pattynama

Department of Radiology,

Erasmus Medical Center,

Dr. Molenwaterplein 40,

3015 GD Rotterdam, The Netherlands

\author{
Abstract To evaluate the use of \\ endovascular coils as markers for \\ respiratory motion correction during \\ high-dose stereotactic radiotherapy \\ with the CyberKnife, an image-guided \\ linear accelerator mounted on a ro- \\ botic arm. Endovascular platinum \\ embolisation coils were used to mark \\ intrapulmonary lesions. The coils \\ were placed in subsegmental pulmo- \\ nary artery branches in close \\ proximity to the target tumour. This \\ procedure was attempted in 25 \\ patients who were considered unsuit- \\ able candidates for standard transtho- \\ racic percutaneous insertion. Vascular \\ coils $(\mathrm{n}=87)$ were succesfully inserted \\ in 23 of 25 patients. Only minor \\ complications were observed: \\ haemoptysis during the procedure
}

(one patient), development of pleural pain and fever on the day of procedure (one patient), and development of small infiltrative changes distal to the vascular coil (five patients). Fiftyseven coils $(66 \%$ of total inserted number) could be used as tumour markers for delivery of biologically highly effective radiation doses with automated tracking during CyberKnife radiotherapy.

Endovascular markers are safe and allow high-dose radiotherapy of lung tumours with CyberKnife, also in patients who are unsuitable candidates for standard transthoracic percutaneous marker insertion.

Keywords Stereotactic radiotherapy · Marker · Lung tumour

\section{Introduction}

Conventional radiotherapy for early stage non-small cell lung cancer (NSCLC) has poor clinical outcome [1]. New radiotherapy techniques have been developed to deliver a higher radiation dose to the lung tumour while sparing adjacent normal tissue [2-6]. Common to these new regimens is the control for respiratory motion in combination with the use of a stereotactic coordinate system [7, 8]. Target localisation can be further improved by the insertion of small metallic markers into the lung tumour, which are automatically detected by the image guidance of tumour tracking radiotherapy systems (TTRS) [8] or internal gating systems [6]. Both marker-based treatment systems enable a considerable reduction of the safety margin to avoid a geometric miss, resulting from tumour motion outside the radiation beam. This limits the volume of nearby healthy tissues that will be irradiated, reducing the toxicity of the treatment. As a result, high biologic effective radiation doses shown to obtain local control rates on the order of $90 \%$ in stage I NSCLC can then be safely administered $[9,10]$.

The standard technique of marker insertion for tumour tracking is a direct percutaneous transthoracic puncture [8]. The insertion of a marker inside the lung tumour was shown to enable an accurate three-dimensional (3D) target localisation during the respiratory motion [6].

However, not all lung tumours (e.g., cavernous tumours or small, lesions without clear contours) are suitable for transthoracic percutaneous marker placement. Furthermore, the $22-45 \%$ risk of pneumothorax after transthoracic marker placement may be unacceptably high for some of 
this generally frail patient population due to severe cardiopulmonary co-morbidity [8,11-13]. Thus, many patients with NSCLC who might potentially benefit from curative radiotherapy currently cannot undergo such high-dose radiotherapy treatment because of the limitations inherent to marker placement by transthoracic puncture.

Therefore, we explored the use of vascular embolisation coils, positioned in small subsegmental pulmonary artery branches surrounding the tumour, as markers for tumour tracking. Endovascular marker placement would be an achievable alternative also in tumours difficult to reach for transthoracic puncture. This would also eliminate the risk of pneumothorax. Here, we report our initial results on the feasibility, effectiveness and complications of endovascular coils, as markers for the Synchrony ${ }^{\mathrm{TM}}$ [14], the respiratory tumour tracking system of the CyberKnife (Accuray, Sunnyvale, CA).

\section{Materials and methods}

Patients

From May 2005 to November 2006, 50 patients with a lung tumour were referred for frameless motion-corrected stereotactic radiotherapy with the Synchrony ${ }^{\mathrm{TM}}$ system of the CyberKnife. In 25 patients, metallic markers for tumour tracking were inserted using direct transthoracic puncture. In 25 other patients (18 male, 7 female), direct transthoracic puncture was considered not feasible or too risky due to: possible major clinical consequences of a pneumothorax for six patients (history of pneumonectomy ( 2 patients), bilobectomy (two, including one patient with a lesion without clear contours), lobectomy (one) and one oxygen dependent patient), a major risk to develop pneumothorax (two patients with one lesion in each lung, including one patient with a cavernous tumour; two patients with two lesions in the same lung), the combination of advanced age and/or cardiopulmonary toxicity (15 patients). The median age of these 25 patients was 79 years (range, 28-89). Twenty patients had primary NSCLC, which was biopsy proven in 12. Five patients were treated for metastatic lung disease: one of cervical carcinoma (two lesions, left-sided and right-sided), two of rectal carcinoma (including one patient with one lesion in each lung), one of malignant melanoma (two lesions in the left lung) and one of a breast carcinoma (two lesions in the right lung). Table 1 shows the diameter, number and localisation of the lung lesion(s). The Charlson Comorbidity Score [15], assessing the comorbidity of a given patient by accounting for several health disorders, is also shown in Table 1. Approval from the Ethics Committee was obtained for the use of vascular embolisation coils as marker for lung tumours, and all patients gave informed consent prior to the insertion of embolisation coils.
Table 1 Charlson Comorbidity Score and tumour characteristics

\begin{tabular}{|c|c|c|c|}
\hline Patient number & $\begin{array}{l}\text { Diameter of } \\
\text { nodule(s) } \\
(\mathrm{mm})\end{array}$ & $\begin{array}{l}\text { Number of } \\
\text { nodules, lung side }\end{array}$ & $\begin{array}{l}\text { Charlson } \\
\text { Comorbidity } \\
\text { Score }\end{array}$ \\
\hline 1 & 36 & $1, \mathrm{R}$ & 3 \\
\hline 2 & $31 / 28$ & $2, \mathrm{~L}$ & 6 \\
\hline 3 & 32 & $1, \mathrm{R}$ & 3 \\
\hline 4 & 44 & $1, \mathrm{R}$ & 5 \\
\hline 5 & 27 & $1, \mathrm{R}$ & 8 \\
\hline 6 & $23 / 27$ & $1 \mathrm{~L} ; 1 \mathrm{R}$ & 6 \\
\hline 7 & 23 & $1, \mathrm{R}$ & 7 \\
\hline 8 & 34 & $1, \mathrm{~L}$ & 1 \\
\hline 9 & 20 & $1, \mathrm{~L}$ & 3 \\
\hline 10 & 50 & $1, \mathrm{R}$ & 1 \\
\hline 11 & 32 & $1, \mathrm{R}$ & 1 \\
\hline 12 & 24 & $1, \mathrm{~L}$ & 3 \\
\hline 13 & 40 & $1, \mathrm{R}$ & 2 \\
\hline 14 & 30 & $1, \mathrm{R}$ & 5 \\
\hline 15 & 29 & $1, \mathrm{~L}$ & 3 \\
\hline 16 & 55 & $1, \mathrm{R}$ & 1 \\
\hline 17 & 26 & $1, \mathrm{R}$ & 2 \\
\hline 18 & $17 / 20$ & $2, \mathrm{R}$ & 6 \\
\hline 19 & $16 / 6$ & $1 \mathrm{~L}, 1 \mathrm{R}$ & 3 \\
\hline 20 & 40 & $1, \mathrm{R}$ & 5 \\
\hline 21 & 30 & $1, \mathrm{R}$ & 2 \\
\hline 22 & 11 & $1, \mathrm{R}$ & 3 \\
\hline 23 & 24 & $1, \mathrm{~L}$ & 4 \\
\hline 24 & 15 & $1, \mathrm{~L}$ & 2 \\
\hline 25 & 29 & $1, \mathrm{R}$ & 3 \\
\hline
\end{tabular}

*Failure to insert coil, $\mathrm{L}=$ left lung, $\mathrm{R}=$ right lung

Technique of endovascular marker insertion

The patient was installed in the supine position, and the procedure was performed in the interventional suite.

A 7-F pulmonary angiography catheter (Grollman-type angled pigtail) was inserted through the femoral vein (although both sides can be used, the right femoral vein was habitually punctured) and positioned in the left or right main pulmonary artery under ECG monitoring (Fig. 1). Over a .035 " guide wire, the catheter was exchanged for a 5-F angled tipped general-purpose catheter with a single end hole. The catheter was advanced over the guide wire into one of the subsegmental pulmonary artery branches in the vicinity of the lung tumour. Larger but not smaller tumours could be well seen with fluoroscopy during the procedure. The position of smaller tumours was inferred from the pre-procedural CT examination of the lungs, and catheter placement was then based on segmental anatomy of the pulmonary arteries. When in the desired position, the catheter was wedged into a small subsegmental pulmonary 


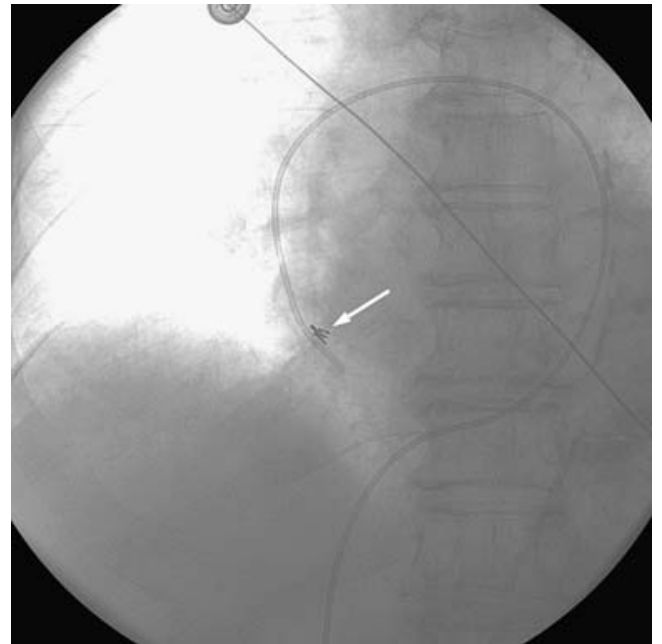

Fig. 1 A pulmonary arterial catheter used to place a coil. The inserted coil is indicated by the arrow: $179 \times 179 \mathrm{~mm}(96 \times 96 \mathrm{DPI})$

artery branch and a 3-4-mm tapered platinum embolisation coil was placed (Tornado Embolization coils, Cook, Bloomington, IN). Platinum coils were used because of their high radio-opacity. Ideally, three coils were placed.

After the procedure, we recorded any clinical symptoms suggestive of immediate pulmonary complications. After an average period of 7 days (range, 2-19 days), a noncontrast-enhanced four-dimensional multislice planning CT (4-D CT) examination was performed, which was evaluated for possible complications of coil placement. The 4-D CT was also used to appreciate the extent of synchronous motion of the tumour with coils on a given distance.

Complications were classified according to the Society of Interventional Radiology (SIR) reporting standards [16]. The SIR standards of the practice committee classifies the complications by outcome: type A complications are minor complications that require no therapy and have no consequence; type B complications are minor complications requiring nominal therapy, including overnight admission, for observation only and have no consequence; type $\mathrm{C}$ complications are major complications requiring therapy and include minor hospitalization $(<48 \mathrm{~h})$; type D complications are major complications that require major therapy, causing an unplanned increase in level of care, and include a prolonged hospitalization $(>48 \mathrm{~h})$; type $\mathrm{E}$ complications are major complications resulting in permanent adverse sequelae, and type $\mathrm{F}$ complications result in death of the patient.

\section{CyberKnife}

The CyberKnife is a frameless image-guided radiotherapy system. The 6-MV linear accelerator is mounted on a robotic arm possessing six degrees of motion freedom. The
CyberKnife therapy unit is equipped with an imageguidance system that consists of two $\mathrm{kV}$ X-ray tubes attached to the ceiling of the treatment room and a pair of orthogonally positioned flat-panel imagers mounted on the floor. A marker extraction algorithm automatically detects the position of the markers in the X-ray images. Basically, the algorithm searches for areas with high contrast in both images that could originate from a single marker. These areas are candidate markers. On the basis of the expected characteristics of the markers and their expected relative positions from the planning $\mathrm{CT}$, the algorithm determines which candidates are true markers and reconstructs the marker locations in 3D. Finally, the 3D marker locations are registered to the marker locations that were determined in the planning $\mathrm{CT}$ to obtain the translations and rotations. Although one marker is sufficient to reduce translational setup errors of the target [17], three markers were required to distinguish marker migration from true tumour motion. As long as the three markers do not migrate in the same direction to the same extent, their migration can be detected by the change in the mutual distance between the markers. The probability that the three markers would migrate an identical distance and direction may be considered sufficiently small to warrant the use of this three marker method for the detection of marker migration.

The Synchrony ${ }^{\mathrm{TM}}$ is the respiratory tumour tracking system of the CyberKnife. The system automatically compensates for the tumour motion by continuously adjusting the position of the beam. The actual motion of the linear accelerator is determined by a correspondence model that relates the respiratory signal, registered by lightemitting diodes placed on the skin, with the internal metallic markers that are localised by the $\mathrm{kV}$ X-ray system. The correspondence model is built at the start of the treatment by acquiring $\mathrm{kV} \mathrm{X-ray} \mathrm{images} \mathrm{at} \mathrm{different} \mathrm{phases}$ of the breathing cycle. During the treatment this model is continuously updated by acquiring X-ray images every 3 to 5 min.

\section{Results}

\section{Coil placement}

Endovascular coils $(n=87)$ were succesfully inserted in 23 of 25 patients in whom the procedure was attempted. A median number of 3 coils for each tumour was inserted (range, 1-5). Table 2 depicts the number of coils for each nodule and the total number of coils for each patient. Coils could also be safely inserted in the two patients with a lesion in each lung as well as in one patient with a medical history of pneumonectomy. In five cases the ideal number of three coils was not inserted due to: haemoptysis during the procedure after insertion of the 2 nd coil $(n=1)$, a centrally located tumour difficult to access $(n=2)$, long duration of the procedure and fatigue of the patient $(n=2)$. 
Table 2 Number of coils inserted per nodule and per patient

\begin{tabular}{lll}
\hline Patient number & Coil per nodule & Total coils inserted \\
\hline 1 & 5 & 5 \\
2 & 3 & 6 \\
3 & 2 & 2 \\
4 & 4 & 4 \\
5 & 3 & 3 \\
6 & $2 \mathrm{R}, 3 \mathrm{~L}$ & 5 \\
7 & $*$ & $*$ \\
8 & $*$ & $*$ \\
9 & 2 & 2 \\
10 & 4 & 4 \\
11 & 4 & 4 \\
12 & 3 & 3 \\
13 & 4 & 4 \\
14 & 4 & 4 \\
15 & 3 & 3 \\
16 & 3 & 3 \\
17 & 3 & 3 \\
18 & 2,3 & 5 \\
19 & $2 \mathrm{R}, 1 \mathrm{~L}$ & 3 \\
20 & 5 & 5 \\
21 & 4 & 5 \\
22 & 3 & 4 \\
23 & 3 & 5 \\
24 & 5 & 5 \\
25 & 3 & 5 \\
\hline
\end{tabular}

*Failure to insert coil, $\mathrm{R}$ = right lung, $\mathrm{L}=$ left lung.

These last two patients included a patient in whom two tumours were marked. In this patient, the procedure was halted after inserting the fifth coil, that is, the second coil for the second tumour.

The procedure time was on average approximately 60 min. All the interventions, including also the patients with multiple lesions, could be performed at once.

The procedure failed in two patients. Here, it proved difficult to negotiate the angiography catheter through the pulmonary valve also whenever the Grollman catheter had been exchanged for a balloon-tipped flow-guided catheter. Catheter manipulation in the right ventricle induced ventricular arrhythmias, which was the reason to terminate the procedure. In both patients, we chose to resort to CTguided transthoracic puncture despite the anticipated risk of pneumothorax rather than proceeding with the endovascular approach and increase the complications risk angiographically by using full narcosis, more extensive protection against arrhythmia and more sophisticated angiography devices including guiding catheters. Trans- thoracic marker placement was successful in both patients, but resulted in the development of a small pneumothorax in one case.

\section{Complications}

There were no immediate procedure-related complications except for one patient who had limited haemoptysis (type A complication), probably caused by wedging the catheter too forcefully in the pulmonary artery end branches during placement of the second coil. The procedure was stopped and the haemoptysis resolved within $10 \mathrm{~min}$ without ensuing clinical sequelae. One other patient, who had two lung metastases from cervical cancer marked, developed chest pain and fever suggestive for pulmonary infarction, on the day of coil insertion (type $\mathrm{C}$ complication). The patient was treated with standard analgetics and was admitted more than 2 days in the hospital to exclude other complications such as pneumonia or pulmonary embolism. The pain was probably pleuritic because two coils were placed against the visceral pleura and caused pleuritic friction and inflammation. All the other patients were discharged from the day-care facilities after a 2-h postprocedural observational period.

During the interval between the coil insertion and the start of the CyberKnife radiotherapy (average 15 days, range, 7-29), none of the patients reported (an increase of) dyspnea or fever, except the single patient with type C complication. The radiation planning CT scan of the lungs showed no procedure-related abnormalities in 18 of 23 patients. In five patients there were small infiltrative changes distal to one of the coils suggestive for small areas of pulmonary infarction (type A complication).

\section{Accuracy of vascular coils as tumour markers}

All patients could be successfully treated with the CyberKnife, based on the recognition of minimally one marker per lesion. Of the 87 coils initially placed, 57 were used as tumour markers for automated tracking during CyberKnife radiotherapy. Coils that were deemed unsuitable markers (see below) were not used by the human operator during treatment.

Twenty-four coils were not used as markers because they did not complete their winding and thus did not have a completely rounded shape. Six coils were disabled based on a distance judged to be too large (range, $56-123 \mathrm{~mm}$ ) to guarantee a similar motion of the coils as the tumour. The median distance between all the inserted coils and the tumour border was $18 \mathrm{~mm}$ (range, $0-123 \mathrm{~mm}$ ). Eighteen coils $(21 \%)$ were directly abutting the tumour. In one patient, none of the coils was in an acceptable position. In retrospect, this was due to erroneous placement of coils in an entirely wrong position based on imaging during 
placement in the anterior-posterior view only. In this patient, satisfactory coil position was achieved in a second angiographic procedure (not counted in this analysis as an index procedure).

It is of note that all inserted coils were detected during planning CT and showed no migration, which means the coils were in a stable, fixed position, during the interval between the planning $\mathrm{CT}$ and the completion of treatment, which was partitioned over the course of several days.

\section{Discussion}

Our results show that endovascular coils can be used as reliable markers for automated tumour tracking during high-dose radiotherapy for lung tumours with the CyberKnife. Markers are placed in the interventional radiology suite by using standard, off-the-shelf angiography equipment and embolisation coils and by using local anaesthesia only. The procedure is well tolerated, safe and may be performed on an outpatient basis. After placement, the coils remain in a stable position and do not migrate as these are wedged in small pulmonary artery branches.

The endovascular route, therefore, is a valuable alternative to the standard method of marker placement by percutaneous transthoracic puncture. The percutaneous method, on the other hand, is associated with a $22-45 \%$ risk of pneumothorax, which requires a chest tube insertion in $27 \%$ of the patients with obstructive lung disease, a particularly frequent disease in this patient population $[8$, 11-13].

Also, the endovascular approach is feasible whenever transthoracic puncture is difficult or not possible whenever the tumour mass is not well defined (e.g., cavernous tumours, Fig. 2) or whenever the lesion is in a hard to reach lung area by direct puncture. By also applying endovascular coils as possible tumour markers, many more patients with lung tumours can now be treated with CyberKnife radiotherapy. This enables the administration of high radiation doses, reported to offer excellent clinical results similar to radical surgery for early stage NSCLC $[9,10$, 18]. Although not specifically adressed in this report, we may expect the use of embolisation coils for marking lung tumours to be of benefit for every radiotherapy system using image guidance to detect markers in order to improve the target localisation.

We did not encounter clinically relevant complications after endovascular coil placement. One patient experienced an episode of transient pleural pain, one patient had shortlived haemoptysis during coil placement, and in two patients we saw clinically silent CT findings suggestive of small pulmonary infarction. This was in accordance with the anticipated complication rate based on previous experience with coil placement in the pulmonary arteries for other indications, notably, pulmonary arteriovenous malformations. Based on this, one would expect to

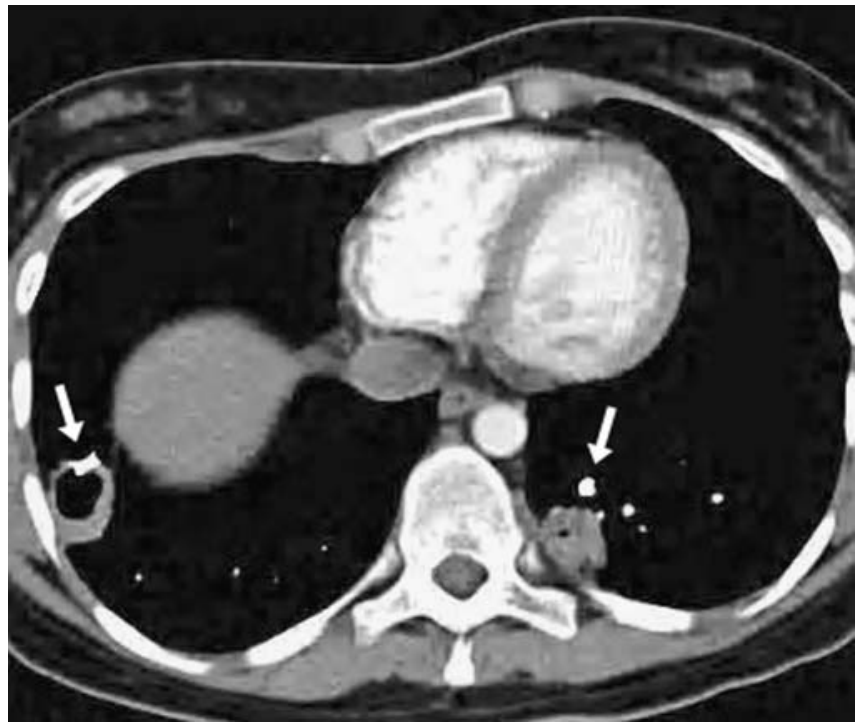

Fig. 2 Patient with two lung metastases (left and right sided) from a cervical carcinoma. Transthoracic marker insertion in the right cavernous lesion is not feasible. Furthermore, transthoracic placement in both lesions would be related to a major risk for pneumothorax. After endovascular marker placement (coils are indicated by an arrow), both lesions could be treated with CyberKnife. $98 \times 83 \mathrm{~mm}(96 \times 96 \mathrm{DPI})$

encounter pulmonary infarction in $6 \%$, pleuritic chest pain in 33\% and groin haematoma in 3\% [19].

Another alternative method for marker placement is the endobronchial placement using video flexible bronchoscopy with a modified transbronchial needle aspiration. The rationale to develop this method was the reduction of the risk of pneumothorax. However, bronchoscopic marker placement is performed under general anaesthesia (44\%) or conscious sedation $(56 \%)$ [20].

While transcutaneously placed Visicoil gold localisation markers (RadioMedCorp., Tyngsboro, MA) were shown to be stable within tumours throughout the treatment duration [21], markers placed under bronchoscopy guidance tend to have less stable fixation. In 3 out of 6 central lung lesions and 3 out of 41 peripheral lesions, Shirato et al. encountered a dropping of the marker out of the lesion [22]. Another study reported that $21 \%$ of the inserted markers could not be detected on the planning CT scan made at $0-5$ days after insertion and probably were coughed up [23]. Furthermore, the variation in the position of bronchoscopically implanted markers exceeded $2 \mathrm{~mm}$ in $9 \%$ of the treatment sessions, necessitating more frequent reexamination with CT scanning. In our study population, no gross migration was observed between the planning CT and during treatment delivery period.

Radiofrequency ablation (RFA) is another treatment recently investigated in the care of medically inoperable lung cancer and was shown to obtain promising results. Simon et al. reported 3-year local control rates of 57\% and $25 \%$ for T1 and T2 NSCLC tumours, respectively [24]. 


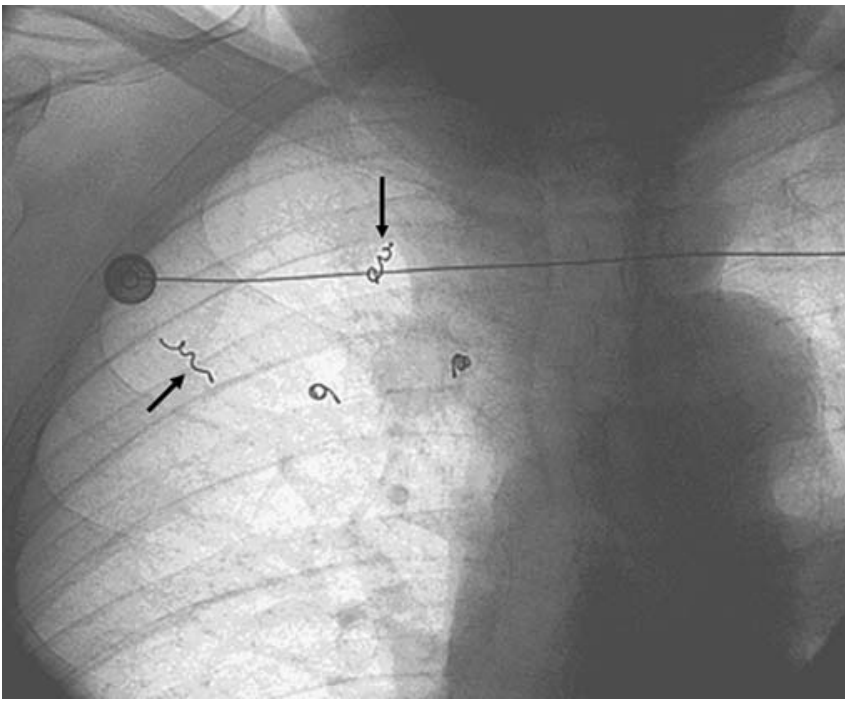

Fig. 3 Non-coiling of the coil because of mismatch between the too large coil windings relative to the small vessel diameter. The coil is therefore unsuitable for automated tracking. The arrows indicate two coils that were unsuitable for tracking due to noncoiling: $137 \times 112 \mathrm{~mm}(96 \times 96 \mathrm{DPI})$

The 36\% overall 3-year survival rate for stage I NSCLC was comparable to those obtained with standard external beam radiation therapy as reported in the review of Qiao et al [25]. The report of Simon highlighted the role of tumour size in the prediction of the local tumour progression rate of tumours smaller than $3 \mathrm{~cm}$ being more effectively treated with RFA than larger tumours. This observation confirmed the encouraging results of previous reports showing complete tumour necrosis in $69 \%-100 \%$ in the tumours

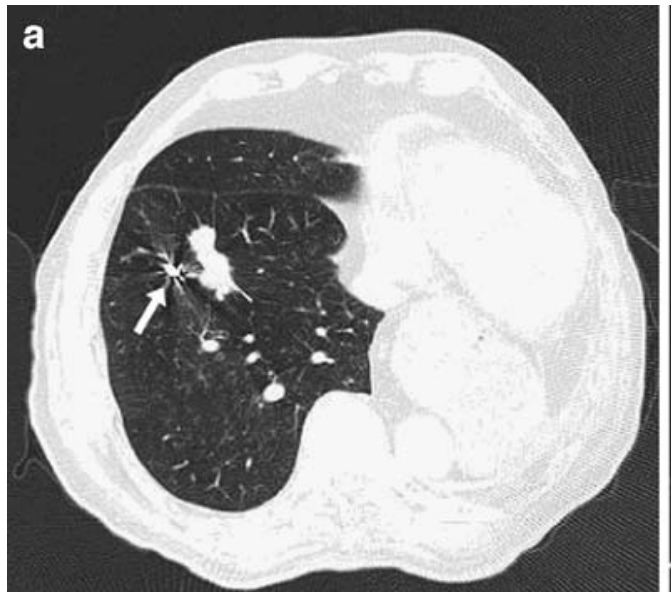

Fig. 4 a Patient with a second primary NSCLC in the right lung, after having undergone left pneumonectomy 5 years earlier for early stage NSCLC. Surgical treatment was considered not feasible because of the previous pneumonectomy. Conventional radiotherapy, using large margins to take tumour motion into account, would result in increased risk for lung toxicity. Considering the low forced expiratory volume in $1 \mathrm{~s}$ of $1,090 \mathrm{ml}$ ( $38 \%$ of predicted), percuta- smaller than $3 \mathrm{~cm}[26,27]$. However, although these results are very promising, these appear to be inferior to the local control rates on the order of $90 \%$ obtained in stage I NSCLC with high-dose stereotactic radiotherapy [10].

Radiofrequency ablation was also shown by Simon et al. [24] to have an acceptable toxicity profile in patients who refused or who were not candidates for surgery with an overall pneumothorax rate of $28 \%$ with $10 \%$ requiring a chest tube insertion. Of concern is the procedure-specific 30 -day mortality rate of $2.6 \%$ (4 of 153 patients). Of note, two of the patients with procedure-related deaths had previously undergone a pneumonectomy. De Baere et al. reported a pneumothorax rate of $54 \%$ with $9 \%$ requiring a chest tube [28]. Similar morbidity data were reported in other RF ablation reports [29, 30]. Randomised trials are required to accurately determine the place of radiofrequency ablation in the treatment of medically inoperable NSCLC patients.

Some limitations of our results need further attention. In this preliminary study we used standard, commercially available platinum embolisation coils of a tapered shape with gradually increasing diameter of the individual coil windings from 3 to $4 \mathrm{~mm}$. These proved to be useful, but not ideal markers for the CyberKnife. In 24 of 87 cases (27\%) the 4$\mathrm{mm}$ diameter windings proved too big for the small subsegmental artery branches in which the angiography catheter was wedged. These windings remained stretched (non-coiling), which rendered these coils unsuitable for automated tracking (Fig. 3). Obviously, smaller winding coils are needed, but still on a .035 " wire-based platform to ensure adequate radio-opacity and for ease of delivery through standard angiography catheters. As a further advantage, smaller coils may allow even more distal insertion

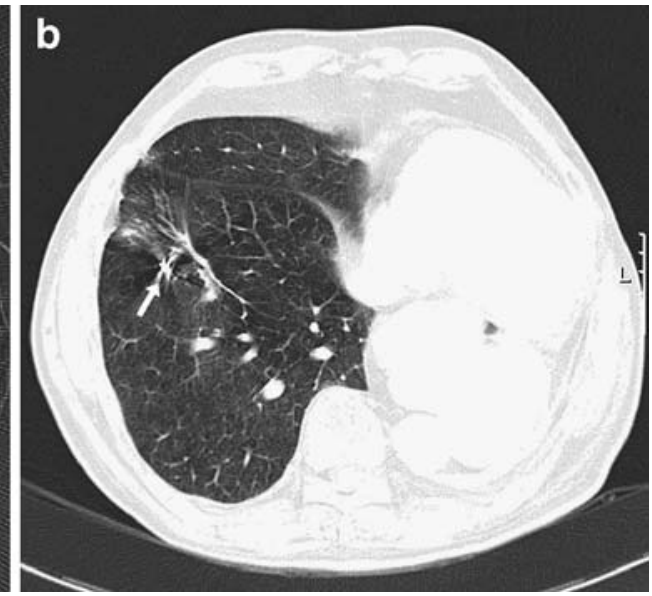

neous insertion of markers with its risk of pneumothorax was considered potentially life-threatening. Endovascular markers were placed (indicated by the arrow) and the patient was treated with CyberKnife by using a total dose of 45 Gy (administered in three treatment sessions). b Follow-up at 10 weeks. Showed complete tumour response. Arrow indicates the coil without remaining tumour): $84 \times 76 \mathrm{~mm}(96 \times 96 \mathrm{DPI})$ 
in the pulmonary artery branches to further reduce the complication rates of pulmonary infarction and pleural pain.

Smaller coils may also be inserted closer to the tumour than larger coils. In our study, six coils could not be used as the distance from the tumour was too large to guarantee a synchronous motion. We used $4 \mathrm{D}$ CT to evaluate the relationship between both motions. A coil-tumour distance smaller than or approximating $40 \mathrm{~mm}$ was shown to be adequate. The smallest coil-tumour distance to be found unacceptable on 4D CT was $56 \mathrm{~mm}$.

Based on our initial results, dedicated coils for marker purposes are currently being designed in close collaboration with the coil manufacturer. With this development we may expect to increase the proportion of coils that can be used during a CyberKnife treatment.
In conclusion, the vascular insertion of coils is a valuable and safe method for marker implantation for lung tumours. This alternative route for placement is also feasible whenever percutaneous (or bronchoscopic) insertion of markers is not possible or undesirable. As a result, many more patients with lung tumours may now benefit from high-dose high-precision radiotherapy with CyberKnife (Fig. 4).

Open Access This article is distributed under the terms of the Creative Commons Attribution Noncommercial License which permits any noncommercial use, distribution, and reproduction in any medium, provided the original author(s) and source are credited.

\section{References}

1. Graham PH, Gebski VJ, Langlands AO (1995) Radical radiotherapy for early nonsmall cell lung cancer. Int J Radiat Oncol Biol Phys 31:261-266

2. Martel MK, Ten Haken RK, Hazuka MB et al (1999) Estimation of tumor control probability model parameters from 3-D dose distributions of nonsmall cell lung cancer patients. Lung Cancer 24:31-37

3. Hanley J, Debois MM, Mah D (1999) Deep inspiration breath hold technique for lung tumors: The potential value of target immobilization and reduced lung density in dose escalation. Int J Radiat Oncol Biol Phys 45:603-611

4. Ohara K, Okumura T, Akisada M et al (1989) Irradiation synchronized with respiration gate. Int J Radiat Oncol Biol Phys 17:853-857

5. Kubo H, Len P, Minohara S (2000) Breathing-synchronized radiotherapy program at the University of California Davis Cancer Center. Med Phys 27:346-353

6. Shirato H, Shimizu S, Kunieda $T$ et al (2000) Physical aspects of a real-time tumor-tracking system for gated radiotherapy. Int J Radiat Oncol Biol Phys 48:1187-1195

7. McGarry RC, Papiez L, Williams M et al (2005) Stereotactic body radiation therapy of early-stage non-small cell lung carcinoma: phase I study. Int J Radiat Oncol Biol Phys 63(4):1010 1015

8. Whyte RI, Crownover R, Murphy MJ et al (2003) Stereotactic radiosurgery for lung tumors: preliminary report of a phase I trial. Ann Thorac Surg 75 (4):1097-1101
9. Onishi H, Araki T, Shirato H et al (2004) Stereotactic hypofractionated high-dose irradiation for stage I nonsmall cell lung carcinoma. Cancer 101:1623-1631

10. Timmerman R, Papiez L, McGarry R et al (2003) Extracranial stereotactic radioablation: results of a phase I study in medically inoperable stage I nonsmall cell lung cancer. Chest 124: 1946-1955

11. Van Sonnenberg E, Casola G, Ho M et al (1988) Difficult thoracic lesions: CTguided biopsy experience in 150 cases. Radiology 167:457-461

12. Kazerooni EA, Lim FT, Mikhail A et al (1996) Risk of pneumothorax in CTguided transthoracic needle aspiration biopsy of the lung. Radiology 198:371-375

13. Cox JE, Chiles C, McManus CM et al (1999) Transthoracic needle aspiration biopsy: Variable that affect risk of pneumothorax. Radiology 212: 165-168

14. Schweikard A, Shiomi H, Adler J (2004) Respiration tracking in radiosurgery. Med Phys 31:2738-2741

15. Charlson ME, Pompei P, Ales K et al (1987) A new method of classifying prognostic comorbidity in longitudinal studies: development and validation. J Chron Dis 40:373-383

16. Omary RA, Bettmann MA, Cardella JF et al (2003) Quality improvement for the reporting and archiving of interventional radiology procedures. Society of Interventional Radiology Standards of Practice Committee. J Vasc Interv Radiol 14(suppl):S293-S295

17. Shimizu S, Shirato H, Kitamura K et al (2000) Use of an implanted marker and real-time tracking of the marker for the positioning of prostate and bladder cancers. Int J Radiat Oncol Biol Phys 48:1591-1597
18. Uematsu M, Shioda A, Suda A et al (2001) Computed tomography-guided frameless stereotactic radiotherapy for stage I non-small cell lung cancer: a 5year experience. Int J Radiat Oncol Biol Phys 51:666-670

19. Prasad V, Chan RP, Faughnan ME (2004) Embolotherapy of Pulmonary Arteriovenous Malformations: Efficacy of Platinum versus Stainless Steel Coils. J Vasc Interv Radiol 15:153-160

20. Reichner CA, Collins BT, Gagnon GJ et al (2005) The placement of gold fiducials for CyberKnife Stereotactic Radiosurgery Using a Modified Transbronchial Needle Aspiration Technique. J Bronchol 12:193-195

21. Kupelian PA, Forbes A, Willoughby TR et al (2007) Implantation and Stability of Metallic Fiducials Within Pulmonary Lesions. Int J Radiat Oncol Biol Phys 69:777-785

22. Shirato $H$, Harada $T$, Harabayashi $T$ et al (2003) Feasibility of insertion/implantation of 2.0-mm-diameter gold internal fiducial markers for precise setup and real-time tumor tracking in radiotherapy. Int J Radiat Oncol Biol Phys 56:240-247

23. Imura M, Yamazaki K, Shirato et al (2005) Insertion and fixation of fiducial markers for setup and tracking of lung tumors in radiotherapy. Int J Radiat Oncol Biol Phys 63:1442-1447

24. Simon CJ, Dupuy DE, Dipetrillo TA et al (2007) Pulmonary radiofrequency ablation: longterm safety and efficacy in 153 patients. Radiology 243:268275

25. Qiao X, Tullgren O, Lax I et al (2003) The role of radiotherapy in treatment of stage I non-small cell lung cancer. Lung Cancer 41:1-11 
26. Lee JM, Jin GY, Goldberg SN et al (2004) Percutaneous radiofrequency ablation for inoperable non-small cell lung cancer and metastases: preliminary report. Radiology 230:125-134

27. Akeboshi M, Yamakado K, Nakatsuka A et al (2004) Percutaneous radiofrequency ablation of lung neoplasms: initial therapeutic response. J Vasc Interv Radiol 15:463-470
28. de Baere T, Palussiere J, Auperin A et al (2006) Midterm local efficacy and survival after radiofrequency ablation of lung tumors with minimum followup of 1 year: prospective evaluation. Radiology 240:587-596

29. Hiraki T, Sakurai J, Tsuda T et al (2006) Risk factors for local progression after percutaneous radiofrequency ablation of lung tumors: evaluation based on a preliminary review of 342 tumors. Cancer 107:2873-2880
30. Yan TD, King J, Sjarif A et al (2007) Treatment failure after percutaneous radiofrequency ablation for nonsurgical candidates with pulmonary metastases from colorectal carcinoma. Ann Surg Oncol 14:1718-1726 